Portland State University

PDXScholar

8-17-2017

\title{
Low Power, Low Data Rate Transmitter for HF Ionospheric Communications
}

Minh-Nhut P. Dang

Portland State University

Follow this and additional works at: https://pdxscholar.library.pdx.edu/honorstheses Let us know how access to this document benefits you.

\section{Recommended Citation}

Dang, Minh-Nhut P., "Low Power, Low Data Rate Transmitter for HF lonospheric Communications" (2017). University Honors Theses. Paper 473.

https://doi.org/10.15760/honors.473

This Thesis is brought to you for free and open access. It has been accepted for inclusion in University Honors Theses by an authorized administrator of PDXScholar. Please contact us if we can make this document more accessible: pdxscholar@pdx.edu. 
Low Power, Low Data Rate Transmitter for HF Ionospheric Communications

\author{
by \\ Minh-Nhut Dang
}

An undergraduate honors thesis submitted in partial fulfillment of the requirements for the degree of

Bachelor of Science

in

University Honors

and

Electrical Engineering

Thesis Adviser

Dr. Richard Campbell

Portland State University 


\title{
Low Power, Low Data Rate Transmitter for HF Ionospheric Communications
}

\author{
Minh-Nhut Dang \\ Maseeh College of Engineering \\ Portland State Univercity \\ Portland OR, United States of America \\ minhdang@pdx.edu
}

\begin{abstract}
This paper primarily focuses on the subject of designing, building, and testing a low power, low data rate transmitter capable of ionospheric communications. The signals transmitted by the quarter watt transmitter built in this project were able to be received up to two miles away from the transmitter. With an operating current of about $11 \mathrm{~mA}$, a typical $12 \mathrm{~V}$ power source of $\mathrm{AA}$ batteries would allow for continuous transmission for over 227 hours.
\end{abstract}

\section{INTRODUCTION}

The $21^{\text {st }}$ century has spoiled us with seemingly endless amounts of power coming from household wall sockets; it is easy to forget that in remote locations or times of distress, we may have nothing more than a little battery or a solar panel to help us communicate with others. In the last 50 years, the world of data transmission and communications have expanded exponentially with the primary focus always being on transmitting more data in a shorter amount of time. This project, however, takes a step back and explores the possibilities of transmitting little amounts of data, with as little power usage as possible, for a prolonged period of time; and with the help of the ionosphere, these signals may possibly be heard from tens if not hundreds of miles away.

Since the main focus of this project is low power and low data rate, our signals will not travel far when communicating in a straight line through various wall and terrain. Therefore, we rely on the physical properties of the ionosphere to help our signal travel far distances. The ionosphere is the atmospheric layer that contains ions as a result of solar radiation. The extra electrons that is present in the $\mathrm{E} \& \mathrm{~F}$ layers of the ionosphere acts as a signal refractor once they are hit by radio waves, making it possible for our signals to bounce off the atmosphere and return to earth. Fig. 1 displays all of the layers of the ionosphere and their distances relative to the ground. Fig. 2 demonstrates the main idea of ionospheric propagation; after the transmitted waves are refracted, they return to earth where earth's surface acts like a mirror and reflects the radio wave back up to be refracted back to the earth again. With multiple refractions and reflections, it is possible for radio waves to travel tens, hundreds, or even thousands of miles by bouncing between the earth and its ionosphere [1].

Due to Morse code being a universal language used among radio operators, a continuous wave $(\mathrm{CW})$ type transmitter was chosen as the configuration of choice over AM or FM. Also, its simplicity also allows for minimal power and less parts in the building process, making the overall project achievable in the allotted time.

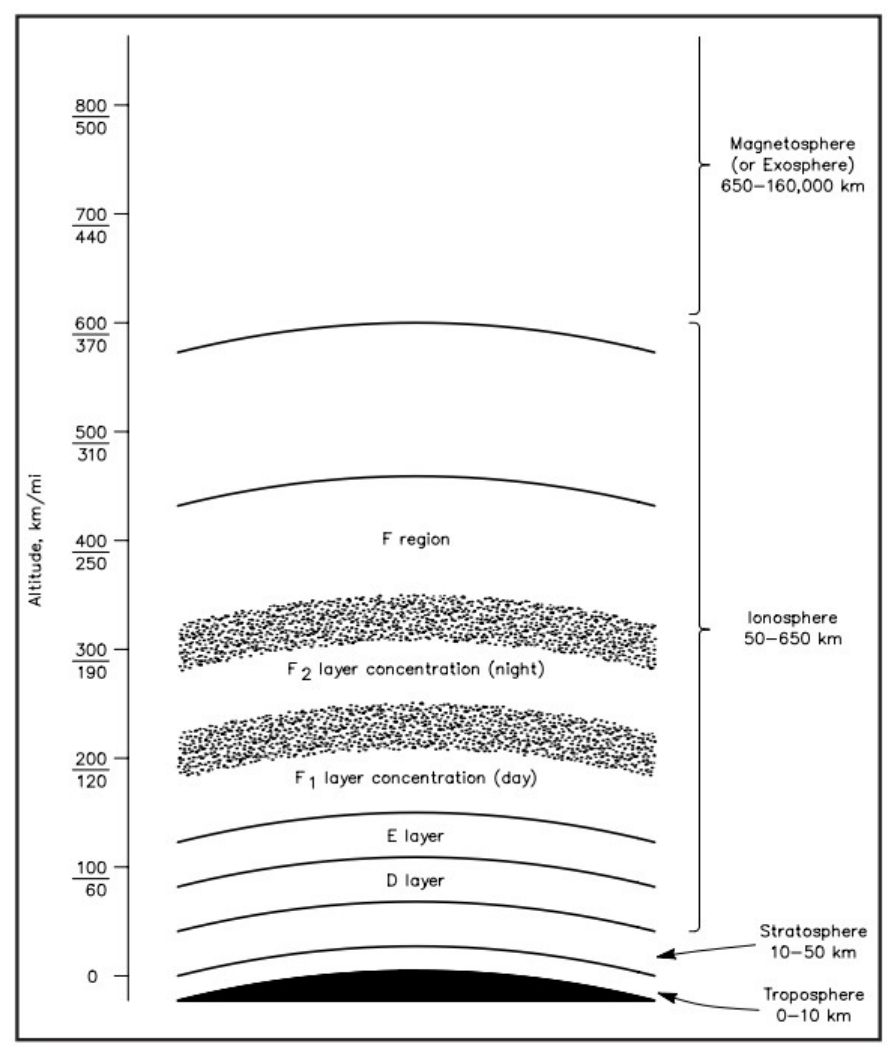

Fig. 1. Areas of the ionosphere, taken from [1] 


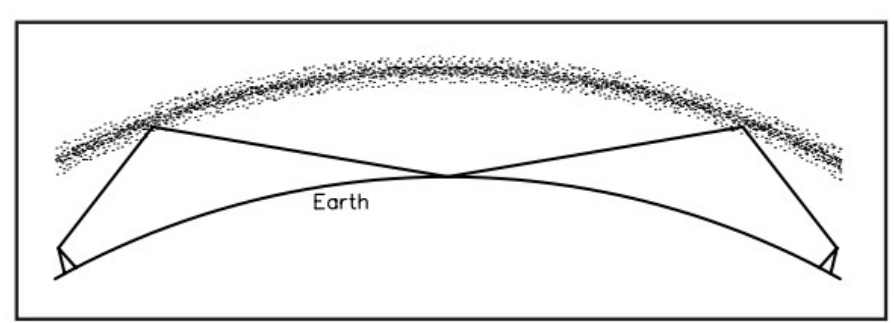

Fig. 2. Multiple signal reflections, taken from [1]

\section{THEORY}

Unlike common amplitude or frequency modulated (AM or FM) transmitters, CW transmitters output unmodulated signals that are simply manipulated by a switch; often called a "key". When pushed down, the switch is closed which allows the circuit to receive power and start transmitting signals. With the key and knowledge of the international Morse code, desired messages can be transmitted through the air with ease. These messages can be anything from an operator's call sign to an SOS message with the operator's coordinates.

To achieve low-data rate, data will simply need to be sent slower than normal. By doing this, the circuit is active for a less amount of time since the transmitter is not consuming any power when data is not being transmitted, thus minimizing power consumption and maximizing battery life. In addition to a low data rate, designing the transmitter to use as little parts as possible will keep our current draw low, thus reducing overall power consumption. A typical $\mathrm{CW}$ transmitter consists of seven parts. These parts and their typical locations within a circuit can be seen in the block diagram displayed in Fig. 3. In a $\mathrm{CW}$ transmitter, there is no need for a modulator like those in AM or FM transmitters, this further reduces parts which help us towards the overall goal of low power. The oscillator creates an electric signal at a desired frequency. That signal is then amplified through some sort of power amplifier, which increases the amplitude of our signal, allowing for better visibility when distinguishing between our Morse code signal and the surrounding noise. The signal is then passed through a keying stage which turns on the circuit when the key is pressed and turns off the circuit once it's released. Finally, the signal is passed through an output filter to narrow down our signal to a narrower bandwidth, allowing the receiver to better tune to the signal without hearing interference from other transmissions. The output of the filtered signal is then passed through an antenna which converts the electric signal into electromagnetic waves that travel through the air.

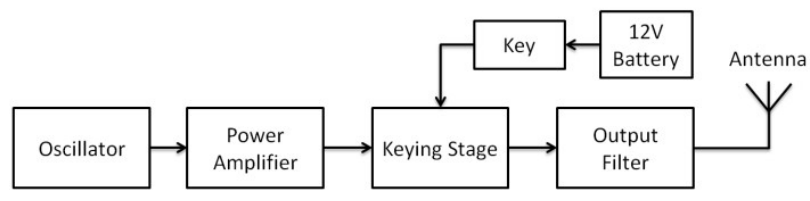

Fig. 3. Basic block diagram of a $\mathrm{CW}$ transmitter

\section{DESIGN}

The overall design of the transmitter was based mainly off of the design of a CW transmitter by Wes Hayward in Experimental Methods in RF Design [4]. This particular design was chosen due to its simplicity. However, after simulating the circuit using LT-Spice, a smaller bandwidth and a higher $\mathrm{Q}$ was desired for the output. To do this, two 7.04 $\mathrm{MHz}$ crystals were utilized to create a half-lattice crystal filter, which gave about the same $-3 \mathrm{~dB}$ bandwidth of $100 \mathrm{kHz}$, but gave a steeper slope outside of the desired band, which will help block out some of the unwanted noise. In real world applications, this is not normally done since crystals are only capable of driving little amounts of power, thus crystal filters are normally placed after a mixer or an oscillator, before the gain stages. This prevents the quartz crystals from being placed through excessive stress and behaving unexpectedly.

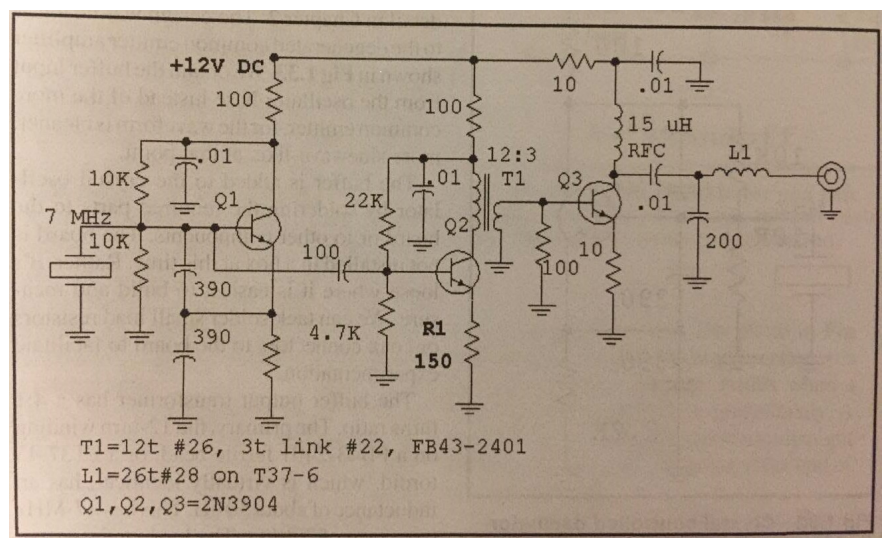

Fig. 4. Wes Hayward's CW transmitter schematic, taken from Fig 1.34 in [4]

The operating frequency of choice for this project was 7.04 $\mathrm{MHz}$. This frequency was chosen due to the nature of $\mathrm{HF}$ signals (between $3-30 \mathrm{MHz}$ ) being known to propagate well through the ionosphere. Furthermore, due to FCC regulations, the $7 \mathrm{MHz}$ band was one of the few $\mathrm{CW}$ bands that my current technician class license allow me to transmit at.

Looking at Fig. 5, the schematic includes, from left to right, a $7.04 \mathrm{MHz}$ crystal connected to an oscillator circuit, which goes into a buffer amplifier with an isolation step-down transformer. The output of the transformer is then attached to the keying circuitry with a class $\mathrm{C}$ driver amplifier which goes through a half-lattice crystal filter and finally out to an antenna.

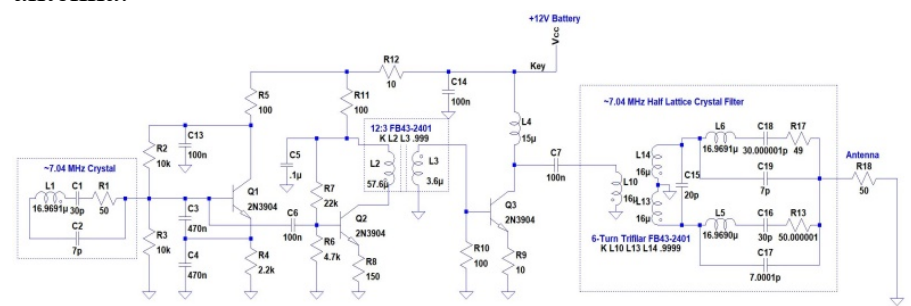

Fig. 5. Final schematic with values used in circuit construction 
Originally, a 40m ferrite rod antenna or a 40m ring antenna was decided on as the two primary antennas for this project. However, due to sourcing complications, a 40m HF mobile antenna seemed like the best alternative. The antenna stands about $7 \mathrm{ft}$ tall, which proved to be quite hard to carry around for prolonged periods of time. All of the parts and devices used for testing and transmitting (aside from equipment found in the lab or at home) are listed in the bill of materials displayed in Fig. 6. Exact prices for these parts were not given since a lot of these parts were sourced locally at a surplus store or Portland State's LID store, thus pricing would not be accurate.

After deciding on all of the parts, the prototype transmitter circuit was assembled on a breadboard. Fig. 7 displays the finalized circuit with the attached key for easy Morse code communication. The antenna connects to a SO239-SMA connector which is then connected to the board. The circuit is powered using a battery pack containing $81.5 \mathrm{~V}$ AA batteries, which provides about 2,500 $\mathrm{mAh}$ of power. With the circuit drawing approximately $11 \mathrm{~mA}$, the battery pack will allow for continuous CW transmission for up to 227 hours. However, since the circuit is designed to be completely off when the key is open, the batteries will last for well over 227 hours when in practical use.

\begin{tabular}{|c|c|c|}
\hline \multicolumn{3}{|c|}{ 7.04 MHZ CW Transmitter Bill of Materials } \\
\hline REF. DESIGNATOR & DESCRIPTION & MFPN \\
\hline Crystals & 7.04MHz 30pF 0C-50C Crystal & LFXTAL019380 \\
\hline $\mathrm{R} 2, \mathrm{R} 3$ & $10 \mathrm{~K} \Omega \pm 5 \% 0.25 \mathrm{~W}$ Resistor & CF14JT10K0 \\
\hline R4 & $2.2 \mathrm{~K} \Omega \pm 5 \% 0.25 \mathrm{~W}$ Resistor & CF14JT2K20 \\
\hline R5,R10,R11 & $100 \Omega \pm 5 \% 0.25 \mathrm{~W}$ Resistor & CF14JT100R \\
\hline $\mathrm{R} 9, \mathrm{R} 12$ & $10 \Omega \pm 5 \% 0.25 \mathrm{~W}$ Resistor & CF14JT10R0 \\
\hline R6 & $4.7 \mathrm{~K} \Omega \pm 5 \% 0.25 \mathrm{~W}$ Resistor & CF14JT4K70 \\
\hline R7 & $22 \mathrm{~K} \Omega \pm 5 \% 0.25 \mathrm{~W}$ Resistor & CF14JT22K0 \\
\hline R8 & $150 \Omega \pm 5 \% 0.25 \mathrm{~W}$ Resistor & CF14JT150R \\
\hline (L2/L3),L4,(L10/L13/L14) & FB43-2401 Toroid & FB-43-2401 \\
\hline $\mathrm{C} 3, \mathrm{C} 4$ & 470nF Capacitor & C322C474M5U5TA \\
\hline $\mathrm{C} 5, \mathrm{C} 6, \mathrm{C} 7, \mathrm{C} 13, \mathrm{C} 14$ & $100 \mathrm{nF}$ Capacitor & C322C104K5R5TA7301 \\
\hline $\mathrm{C} 15$ & 20pF Capacitor & S200K25SL0N63L6R \\
\hline Q1,Q2,Q3 & 2N3904 Transistor & $2 \mathrm{~N} 3904 \mathrm{BU}$ \\
\hline Transmit Antenna & MFJ-1640T 40 Meter HF Stick Mobile Antenna & MFJ-1640T \\
\hline SDR Kit & Software Defined Radio with Antennas & RTL2832U \\
\hline Morse Code Key & Eisco Labs Contact Key & PH0986A \\
\hline
\end{tabular}

Fig. 6. Complete BOM for built transmitter and test setup

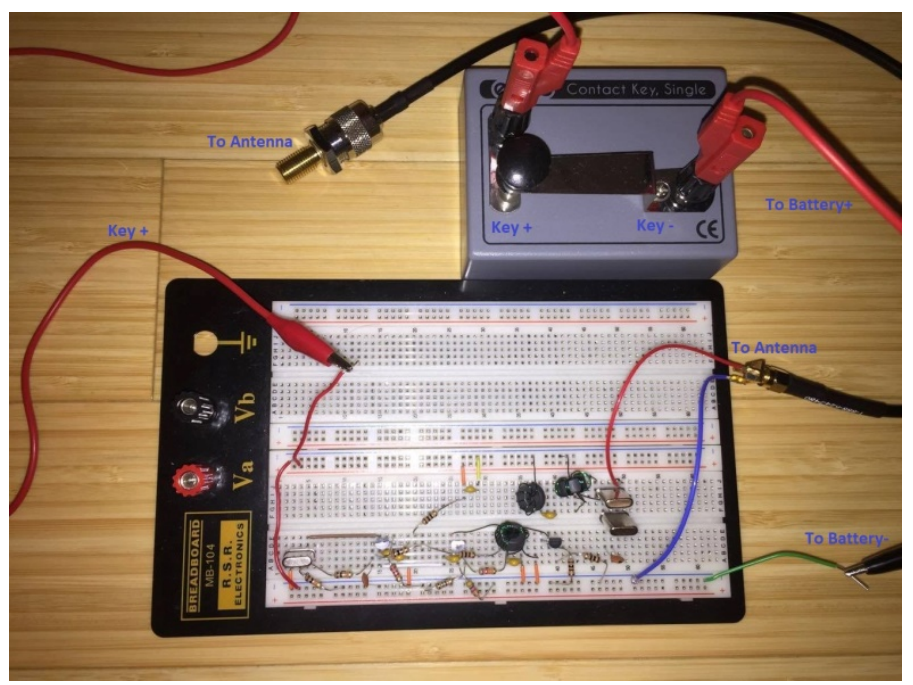

Fig. 7. Assembled CW Transmitter from schematic in Fig. 5

\section{RESULTS}

To test the transmitter, the R820T2 RTL2832U SDR (Software Defined Radio) was used. With Airspy's SDR\# software, the $40 \mathrm{~m} \mathrm{CW}$ band was able to be observed using a $1.5 \mathrm{~m}$ telescopic antenna attached to a magnetic mount. With SDR\# running, the transmitter was taken to $1,2,3$, and 5 miles away from the receiver setup. Fig. 8 displays the receiver setup used to test the transmitter. Due to having only one operator, the SDR receiver was left running and the activities recorded via screen capture software. Meanwhile, the transmitter was taken to various distances away from the location of the receiver (before transmitting over the $40 \mathrm{~m}$ band, it must be noted that a technician class amateur radio license must be obtained; transmission without a license is illegal under FCC regulations. Transmissions in this project was done under my call sign KI7OZP, identifying call signs were not used during the preliminary short transmissions of this project but should be used in any future testing). At each location, the circuit was keyed randomly several times and the time of keying was recorded. With the time recording and the transmissions complete, the receiver data can be analyzed by matching the recorded time with the system time displayed on the computer.

The transmitter worked well when practically next to the receiving antenna. However, when moving further than a few miles, the signal was unable to be detected. The most successful attempt with the transmitter 2 miles away from the receiver is displayed in Fig. 9, where the dots were able to be found well against the noise floor and showed up fairly nicely in the waterfall view. The displayed frequency of 7.039.881 was tuned in by the receiver to achieve the clearest tone.

Due to the transmitter antenna not being fixed, it was hard to achieve a consistent signal when recorded on the SDR. At three and five miles, the signals were not able to be received. It is suspected that this was due to either the angle of the antenna or the power of the antenna. Since the antenna does not output much power, it is possible that the signal just got lost within the noise floor. However, the wide angles of the antenna (relative to vertical), may have resulted in a much wider dead zone. This means that the signals may have been received by a faraway receiver, but the receivers from a few miles away will not be able to pick up the signal since the angle of incidence against the ionosphere is much wider.

As of currently, only tests with spurious random dots have been transmitted over the air with this transmitter. Even so, at these ranges, it is hard to tell whether the transmitter is truly capable of ionosphere transmission or if the signals received are from direct saves from the transmitter. To find out how far these signals can travel, an automatic key would need to be implemented to transmit my call sign over the air repeatedly while I listen for any responses from other operators. However, preliminary tests of this transmitter hold promising results because the signals that were able to successfully be received were very clear and distinguishable. 


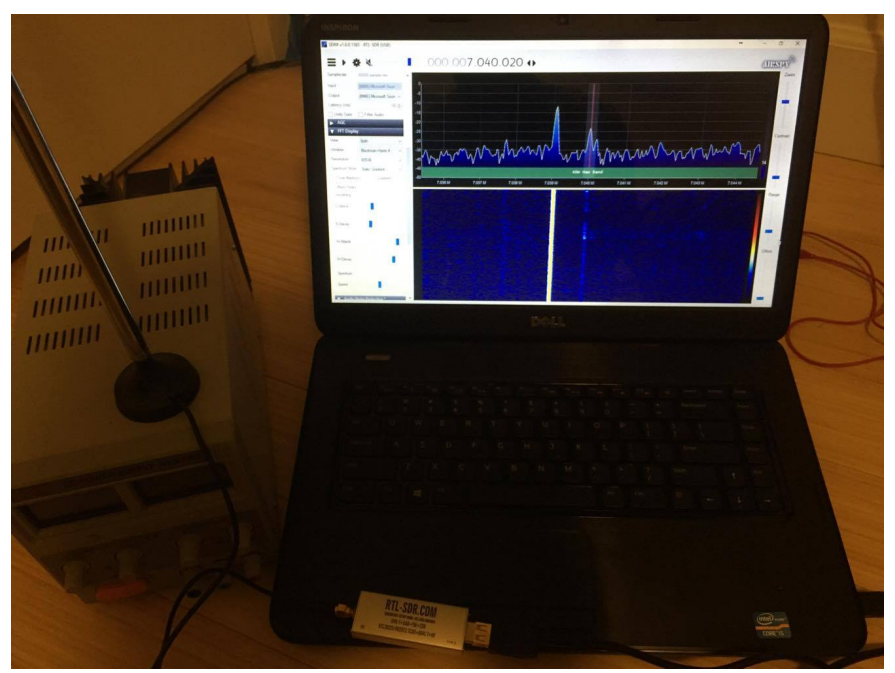

Fig. 8. SDR receiver test setup

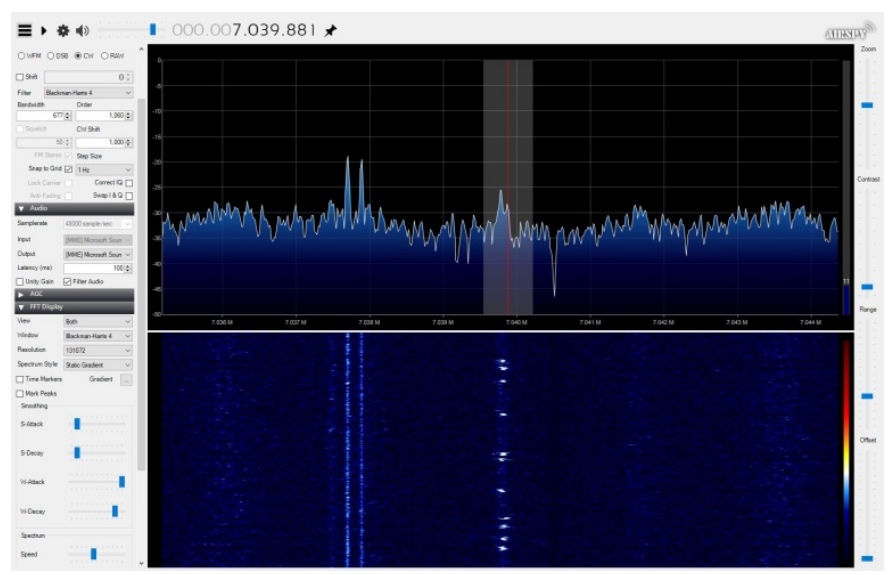

Fig. 9. Dots detected from SDR 2 miles away

\section{CONCLUSION}

The low-power, low data rate transmitter built in this project proved to be working. However, much more extensive and controlled testing still needs to be done before knowing for sure the capabilities of this transmitter in ionospheric communications. There are still many modifications and improvements that can be made to this transmitter prototype to be more efficient and portable. In its current configuration, the transmitter is not viable to be carried around and used in any practical way. However, it is able to act as a transmitting station which would allow for further exploration of ionospheric communications by continuously sending out signals and seeing the farthest receiver station it is able to reach.

There are several changes that will be made to this transmitter in the future in an attempt to improve the overall performance as well as make it portable and small enough for practical use as a transmitter or an emergency beacon. The first modification would be to explore additional filter designs in an attempt to further narrow bandwidth. The next change would be to recreate this circuit on a printed circuit board. I am confident that this transmitter circuit will easily be able to fit into a small PCB that can be placed in a weather-proof box for emergency uses. Furthermore, due to the little amount of power that this circuit consumes, I would like to explore the possibilities of powering the device using a solar cell. I believe that with a small solar cell and a battery on an average sunny day, this device will have no problem powering itself. Another area of improvement could be in the antenna itself. Being the primary if not most important part of a transmitter, different antenna arrays such as a ferrite rod or a ring antenna may very well be the key to achieving a better transmission.

With the current results and changes in mind, there is a clear path for continuous improvement over time on this transmitter. Ionospheric communication is definitely possible and was demonstrated with this transmitter, although at a very small distance. With additional testing and some addition modifications, ionospheric communications over hundreds or thousands of miles continue to be the main goal for this transmitter.

\section{REFERENCES}

[1] I. Poole, "Radio Waves and the Ionosphere," Nature, vol. 154, no. 3909, pp. 413-413, 1944.

[2] C. Luetzelschwab, "The Sun, The Earth, The Ionosphere."

[3] P. Horowitz and W. Hill, The Art Of Electronics. 2015.

[4] W. Hayward, R. Campbell, and Bob Larkin, Experimental Methods in RF Design. 2016.

[5] W. Hayward, "An Updated Universal QRP Transmitter," no. April, pp. 28-32, 2006.

[6] G3XBM, "Ferrite Rod TX Antennas," 2013. [Online]. Available:

https://sites.google.com/site/g3xbmqrp3/antennas/ferrite_tx.

[7] Doug DeMaw, "The Basics of Transmitters." 1984.

[8] W. Clay Laster, The Beginner's Handbook of Amateur Radio. 2001.

[9] P. Pagani, R. Fleury, Y. Le Roux and D. Le Jeune, "A study of HF transmitter geolocation through single-hop ionospheric propagation," The 8th European Conference on Antennas and Propagation (EuCAP 2014), The Hague, 2014, pp.2689-269. 